\title{
Metabolite analysis for the comparison of irrigated and non-irrigated field grown tomato of varying genotype
}

\author{
Yaniv Semel, ${ }^{\mathrm{a}}$ Nicolas Schauer, ${ }^{\mathrm{b}}$ Ute Roessner, ${ }^{\mathrm{b}}$ Dani Zamir, ${ }^{\mathrm{a}}$ and Alisdair Robert Fernie ${ }^{\mathrm{b}, *}$ \\ ${ }^{a}$ The Otto Warburg Center for Biotechnology, Faculty of Agriculture, Food and Environmental Sciences, The Hebrew University of Jerusalem, \\ 76100, Rehovot, Israel \\ ${ }^{\mathrm{b}}$ Max-Planck-Institut für Pflanzenphysiologie, Am Mühlenberg 1, 14476, Potsdam-Golm, Germany
}

Received 11 January 2007; Accepted 28 March 2007

\begin{abstract}
Every year the consequences of water deficit on crop yield and quality are profound. The observation that many wild species relatives of cultivated crops display a greater stress tolerance and the fact that the cultivated species generally display only a fraction of the allelic diversity available within the tomato clade suggest that crossing of wild species with elite cultivars could improve the stress physiology of modern crops. To assess this from the basis of chemical composition we applied an established GC-MS based metabolite profiling method to fruits from irrigated and non-irrigated tomato plants either of the cultivated tomato (Solanum lycopersicum) or of its hybrid with its wild species relative (Solanum pennellii). Results are discussed in terms of both the metabolic response to drought stress and the potential of utilizing exotic germplasm as a means to improve agronomically important characteristics of crop species.
\end{abstract}

KEY WORDS: S. lycopersicum; S. pennelli; water stress; transgenesis; plant breeding; GC-MS metabolite profiling.

\section{Introduction}

Plant evolution under domestication has lead to increased productivity, but at the same time it narrowed the genetic basis of crop species (Zamir, 2001). Natural biodiversity is an under-exploited sustainable resource that can enrich the genetic basis of cultivated plants with novel alleles that improve productivity and adaptation. A major challenge in modern breeding is to return to the wild ancestors of crop plants and employ some of the diversity that was lost during domestication for the improvement of agricultural yields under optimal as well as stress conditions such as those found in the field. Metabolite profiling is being extensively utilized to gain both diagnostic and mechanistic insight into plant responses and adaptations to a wide range of stresses including, but not limited to nutrient deficiency (Urbanczyk-Wochniak et al., 2005), mineral toxicity (Roessner et al., 2006), temperature and oxidative stresses (Kaplan et al., 2004) and osmotic stress (Gong et al., 2005). As a first study aimed at understanding the chemico-genetic basis of the different stress tolerances exhibited by wild species tomato we recently performed a broad range GC-MS metabolite profiling of species of the tomato clade (Schauer et al., 2005). Some of the metabolic variance observed in these species indicates that they could be useful breeding stock for engineering stress tolerance. In order to enhance the rate of progress

\footnotetext{
* To whom correspondence should be addressed.

E-mail: fernie@mpimp-golm.mpg.de.
}

of exotic introgression breeding a population of tomato segmental introgression lines (ILs) comprised of markerdefined genomic regions taken from the drought tolerant wild species $S$. pennellii and introduced into the genetic background of an elite inbred variety was created some years ago (M82; Eshed and Zamir, 1994; 1995). In the mean time these lines have been extensively analysed at the level of various important quality parameters including yield associated traits such as fruit weight, harvest index, seed per fruit etc. (Gur et al., 2004a; Semel et al., 2006), plant architecture (Fridman et al., 2002), leaf morphology (Holtan and Hake, 2003), fruit morphology (Gur et al., 2004b; Semel et al., 2006), fruit pigmentation (Liu et al., 2003) and fruit metabolite content (Schauer et al., 2006).

Much research over the past few years has been focussed on the development of drought tolerant plant genotypes (Hsieh et al., 2002; Kalifa et al., 2004; Bartels and Sunkar, 2005). There is considerable interest in enhancing drought tolerance in crops and in understanding the mechanisms by which plants naturally respond to osmotic stress (Grainer et al., 2005; Bohnert et al., 2006; Mittler 2006; Yamaguchi-Shinozaki and Shinozaki, 2006). Although yield of lines carrying the $S$. pennellii introgressions increased dramatically in nonirrigated trials compared to the control genotype, the final yield was considerably lower than that obtained in the irrigated treatment (Gur and Zamir 2004). Here we evaluate the metabolite content of pericarp of tomato fruit from M82 and its interspecific hybrid with $S$. pennellii under irrigated and non-irrigated 
conditions. We evaluate the degree of metabolic variance due to genotypic and environmental influences and additionally compare the magnitude of changes observed in this study with that we have previously characterized in greenhouse grown transgenic tomato lines. Finally, data are discussed in the context of current models of drought stress tolerance.

\section{Materials and methods}

\subsection{Chemicals}

All chemicals and pure standard substances were purchased from either Sigma-Aldrich Chemie $\mathrm{GmbH}$ (Deisenhofen, Germany) or Merck KGaA (Damstadt, Germany).

\subsection{Plant growth and harvesting}

Plants were grown in Akko, Israel and were handled largely as described in the literature (Schauer et al., 2006). In detail seedlings (35 days old) were transplanted in the field with $50 \mathrm{~cm}$ between plants and $2 \mathrm{~m}$ between rows $\left(1\right.$ plant per $\left.\mathrm{m}^{2}\right)$. All the plants were sprinkler irrigated immediately after transplanting with $30 \mathrm{~m}^{3}$ of water for every $1000 \mathrm{~m}^{2}$ of field area. For the rest of the growing period the wet treatment was dripirrigated with $320 \mathrm{~m}^{3}$ of water per $1000 \mathrm{~m}^{2}$ throughout the growing season according to the irrigation protocols in the area, while no water was applied to the dry treatment. For each set of samples biological replication of six fruits was used.

\subsection{Metabolite extraction and derivatization}

Tomato pericarp tissue was extracted in $4500 \mu$ of water/methanol (1:2) as described in Roessner-Tunali et al. (2003); $50 \mu \mathrm{l}$ of internal standard (2 $\mathrm{mg}$ of ribitol per $\mathrm{ml}$ ) was added for quantification. All other details were exactly the same as in Roessner et al., (2001).

\subsection{GC-MS analysis and chromatogram evaluation}

The gas chromatography mass spectrometry (GCMS) system was composed of an AS 2000 autosampler, a GC 8000 gas chromatograph and a Voyager quadrupole mass spectrometer (ThermoFinnigan, Manchester, UK). The mass spectrometer was tuned according to the manufacturérs recommendations, using tris-(perflurobutyl)-amine (CF43). GC was performed on a $5 \mathrm{MS}$ column (Varian Deutschland GmbH, Darmstadt, Germany), using the exact same conditions defined in Roessner et al. (2001). Substances were identified by comparison to mass spectral tags represented in our in-house database (Kopka et al., 2005; Schauer et al., 2005b). They were quantified following the same procedure as described in Roessner et al. (2001), the recovery of small representative amounts of each metabolite through the extraction, derivatization, storage and quantification process has been documented previously (Roessner et al., 2000), as has the validity of this method for use with tomato derived extracts (Roessner-Tunali et al., 2003).

\subsection{Statistical analysis}

Principal component analysis (PCA) on the $\log 10$ normalized dataset was performed with the TIGR MultiExperiment Viewer 4.0 (Saeed et al., 2003). Missing values were imputed using the inbuilt KNN algorithm of TMev 4.0 with default settings (Theilhaber et al., 2002). If two observations are described in the text as different, this means that their difference was determined to be statistically different $(p \leq 0.05)$ by the performance of the students $t$-test using the algorithm embedded into Microsoft Excel, differences at the $p \leq 0.01$ level are additionally demarcated within the Supplementary data set (Microsoft, Seattle, WA). The less stringent level was used predominantly since this is more or less standard with respect to plant physiology, however, in light of recent reports on metabolite data sets (see for example Broadhurst and Kell, 2006), we additionally chose to display the results at a more stringent cut off for reference at the readers discretion.

\section{Results and discussion}

\subsection{Morphology of tomato plants of M82 and its interspecific hybrid with $S$. pennellii under irrigated and non-irrigated conditions}

In order to be able to evaluate metabolite composition within the context of whole plant and stress physiological we first evaluated morphological parameters of the parental lines and of the F1 hybrid that resulted from crossing them. Figure 1 shows that as would be anticipated that under good irrigation fruit of the hybrid was intermediate in size and coloration between the large oval-shaped red fruited $S$. lycopersicum and the small green fruited wild species $S$. pennellii. Under field conditions the total plant weight of the F1 hybrid was much greater than that of either parent (note the $S$. pennellii was excluded from this trial since it does not set fruits under the field conditions employed) and for both genotypes irrigated plants had a much higher biomass than non-irrigated plants. In contrast, the fruit yield of the F1 hybrid was less affected by the lack of irrigation than its M82 parent. Interestingly, using a refractometer the total soluble solids (or Brix) content of the fruit was markedly higher in the F1 hybrid than in the elite cultivar and was also less responsive to drought stress (figure 2). 


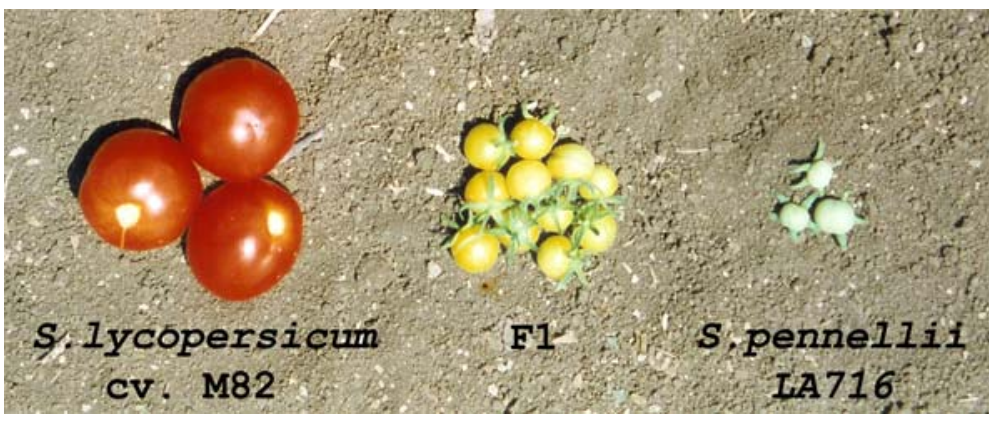

Figure 1. Tomato fruits of S. lycopersicum (cv M82), S. pennellii (Accession LA716) and their interspecific hybrid (F1).

\subsection{Metabolite profiles of tomato plants of M82 and its interspecific hybrid with $S$. pennellii under irrigated and non-irrigated conditions}

Tomato pericarp samples were harvested, extracted and analysed as previously defined (Roessner-Tunali et al., 2003; Schauer et al., 2006). A total of 72 metabolites of known identity were detected using a combination of those metabolites determined using our previous method and our recently established mass spectral databases (Kopka et al., 2005; Schauer et al., 2005). A large amount of metabolic variance was observed in the experiment. For ease of discussion we will begin with a point by point analysis at three different levels (i) comparison of the metabolite composition of the M82 parent and the F1 hybrid of its cross with $S$. pennellii under water replete conditions (ii) comparison of the metabolite composition of the elite cultivar under water replete and deficient conditions and (iii) comparison of the metabolite composition of the F1 hybrid under water replete and deficient conditions. Selected metabolites of particular influence are plotted in figure 3.

Comparison of the two genotypes under water replete conditions gave results that could perhaps have been anticipated from previous studies in which the metabolite levels of fruit pericarp of either S. lycopersicum and $S$. pennellii (Schauer et al., 2005) or the introgression line population resulting from the crossing of these species (Schauer et al., 2006) were investigated. Amino acid levels showed considerable variance between the genotypes with alanine, asparagine, methionine, phenylalanine, serine, threonine and valine all decreasing to levels between 8 and $49 \%$ of those observed in the elite cultivar. In contrast, the majority of fatty and organic acids increased in the F1 hybrid relative to the S. lycopersicum control. The increase in fatty acids being 1.65 to 1.8 -fold, whilst that in organic acids was much greater, reaching as much as 11 -fold in the case of malate. These large changes are surprising in light of our previous study which encompassed analysis of fruit pericarp of S. lycopersicum and S. pennellii since the acid content in both parents was remarkably similar (Schauer et al., 2005). That said the range in the content observed in organic acids in the $S$. pennellii introgression population was similar to that seen for the other metabolites and clear examples of transgressive behaviour were also observed in this population (Schauer et al., 2006). Analysis of the levels of sugars, sugar phosphates, sugar
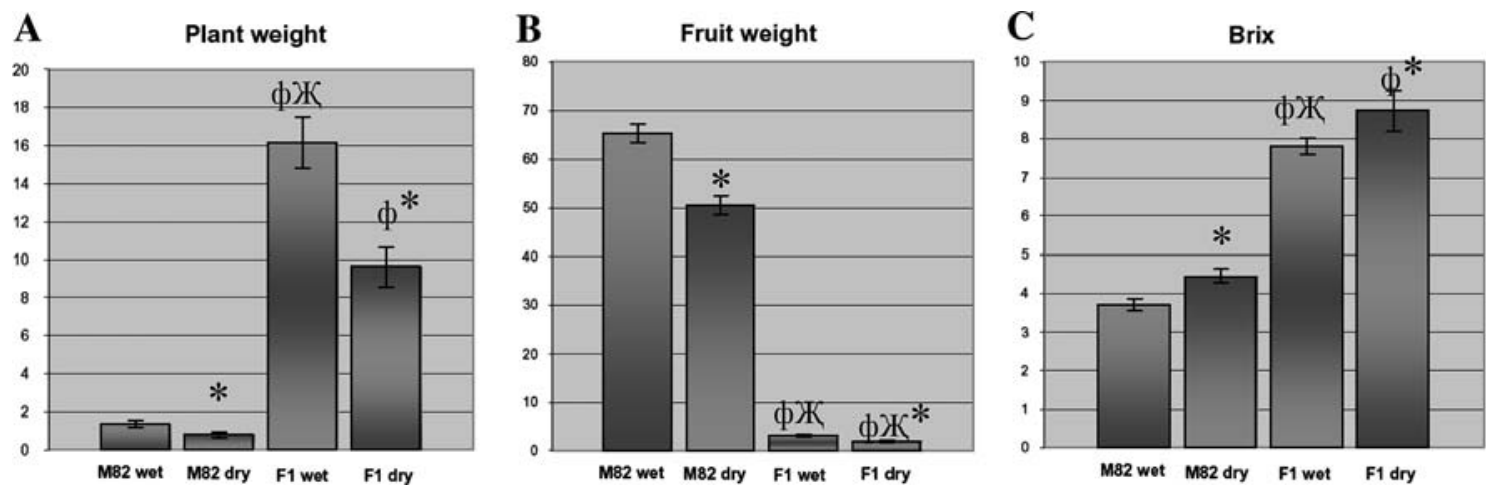

Figure 2. Phenotypic measurements of M82 and its interspecific hybrid with S. pennellii (F1) under irrigated (wet) and non-irrigated (dry) conditions. (A) plant weight (kg), (B) Average fruit weight per plant (g), (C) Average Brix per plant (\%). Values are presented as mean \pm standard deviation of determinations on seven individual plants. Statistical differences between samples were assessed by the Students $t$-test $(p \leq 0.05)$ and are demarcated as follows: ${ }^{*}$ Indicates significant differences between different treatments of a given genotype, $\phi$ indicates significant differences between genotypes under the same irrigation conditions $\mathbb{K}$ indicates significant diffences between non-irrigated M82 and the irrigated F1. 

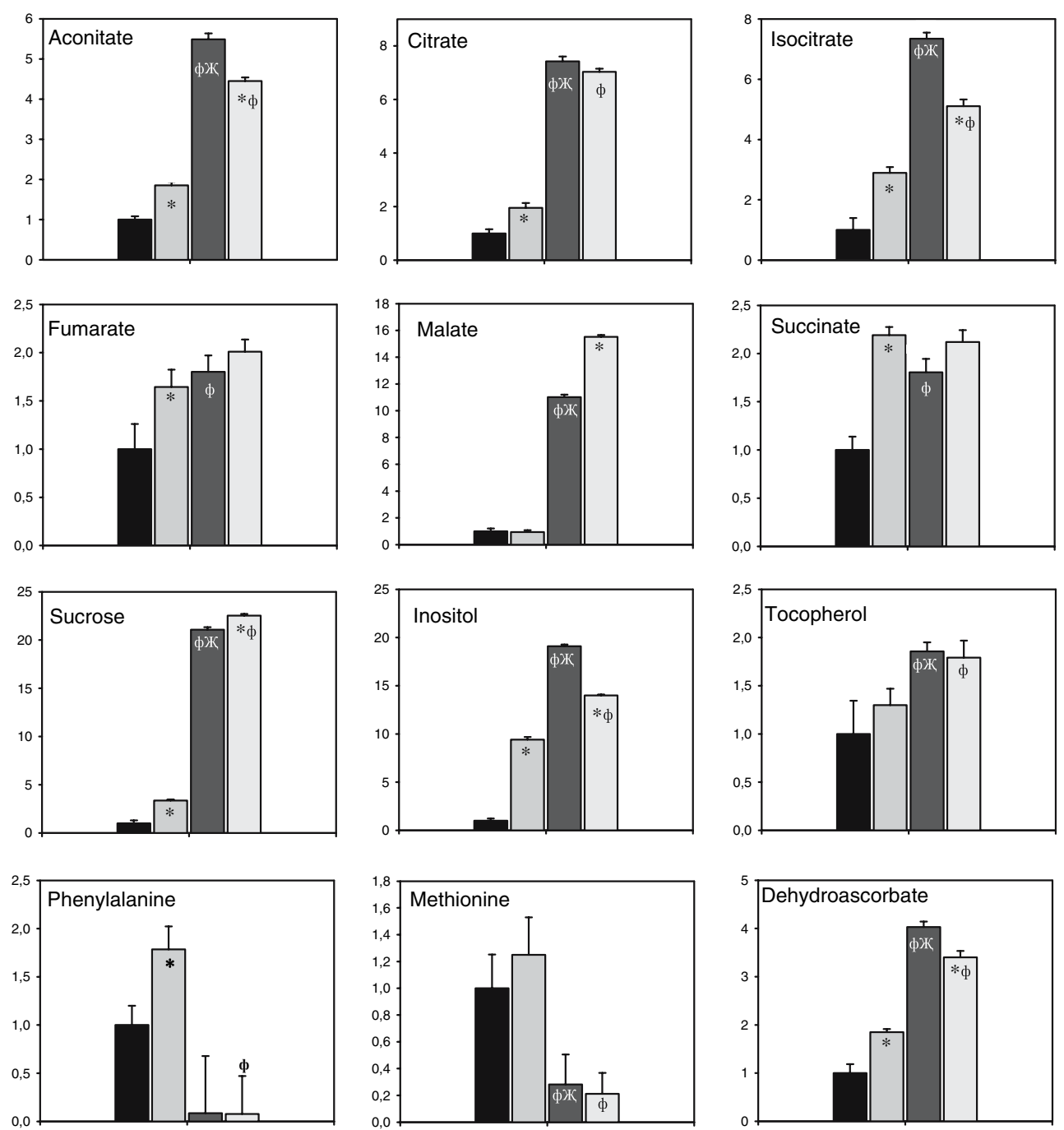

Figure 3. Relative metabolite content of pericarp of tomato fruit from M82 and its interspecific hybrid with S. pennellii under irrigated and nonirrigated conditions. Metabolites were determined as described in "Materials and Methods". The full data set from these metabolic profiling studies can be accessed at our website: http://www-en.mpimp-golm.mpg.de/03-research/researchGroups/01-dept1/Central_Metabolism. Data are normalised with respect to the mean response calculated for the irrigated M82 conditions. Values are presented as mean \pm SE of determinations on six individual plants per line. Statistical differences between samples were assessed by the Students $t$-test $(p \leq 0.05)$ and are demarcated as follows: * indicates significant differences between different treatments of a given genotype, $\phi$ indicates significant differences between genotypes under the same irrigation conditions $\mathcal{W}$ indicates significant diffences between non-irrigated M 82 and the irrigated F1. Black bars denote irrigated M82, light grey non-irrigated M82, dark grey the irrigated F1 and mid-grey the non-irrigated F1.

alcohols and miscellaneous metabolites revealed that the genotypes were quite distinct from one another with respect to their contents of these metabolites also. In general there was a tendency for these metabolites to be elevated in level in the F1 hybrid. Fructose, gentiobiose, glucose, maltose, melizitose, ribose, sucrose, trehalose, xylose and myo-inositol were all present at considerably higher levels in the hybrid with putrescine and fructose 6-phosphate being present at significantly lower levels than in M82.

Comparison of S. lycopersicum under irrigated and non-irrigated plots in the same field also revealed large changes in metabolite contents. In this case, however, more dramatic changes in amino acid content were observed with large increases in alanine, aspartate, $\beta$-alanine, GABA, glutamate, glycine, homoserine, isoleucine, proline, serine and valine, with decreased observed for cysteine, glutamine and glycine. Of these amino acids there is an abundant literature suggesting a role of proline in response to water stress (reviewed in Bartels and Sunkar, 2005; Zhu, 2000), however, whilst this also explains the increase in glutamate which is a precursor in the predominant pathway of proline synthesis (Yoshiba et al., 1997) and the documented increase in glycine could reflect accumulation of another commonly mentioned compatible solute - glycine betaine (Rhoades and Hanson, 1993), the majority of these changes have not been described before. The increase in the branched chain amino acids is intriguing, however, our understanding of the exact role and regulation of 
these metabolites in plants is currently somewhat lacking (Ishizaki et al., 2005). In addition to these changes the levels of fatty and organic acids generally increased with particularly large increases in intermediates of the TCA cycle but a decrease in the level of citramalate (however the function of this metabolite in plants is very poorly understood). An increased activity of the TCA cycle under conditions of osmotic stress has not been reported per se, however, it is known that this stress often also produces oxidative stress which is intimately associated with mitochondrial metabolism as well as being necessary for the assimilation of nitrate that is essential for the production of glutamate (Fernie et al., 2004). There are also large changes in many sugars and sugar derivatives including erythritol, fructose, glucose, isomaltose, sucrose, ribose and trehalose and the oligosaccharide gentiobiose (Dumville and Fry, 2002). With the exception of that in gentiobiose the changes in these solutes have largely been documented previously (see for example Dancer et al., 1990; Büssis and Heineke, 1998; Avelange-Macheral et al., 2006), and the role of sugar accumulation under conditions of water stress is now firmly established (Bartels and Sunkar, 2005). The change in gentiobiose is intriguing, particularly given its proposed signalling role during tomato fruit development (Dumville and Fry, 2002), however, further experimentation is clearly required before a functional role for the change in the level of this metabolite can be defined.
In contrast comparison of the F1 hybrid under irrigated and non-irrigated plots in the same field revealed very few changes in metabolite contents. These were restricted to the large decrease in Pro, isocitrate, maltose, fructose, and nictonate content and increases in mannitol content in the non-irrigated plants. This suggests that if the metabolite levels are contrasted to those of the S. lycopersicum under irrigated conditions the changes observed would be broadly similar. For this reason we also analysed this meta-comparison statistically. With the exception of citramalate, glycolate and quinate (for which only the irrigated F1 hybrid was significantly different from the wild type) and uracil, maltose, and rhamnose (for which only the non-irrigated F1 hybrid was significantly different from the wild type), the changes in metabolite level in the F1 hybrid were broadly similar irrespective of water supply (see figure 3 and Supplementary data).

\section{Concluding remarks}

Having performed detailed point-by-point comparison of the data we wanted to perform a more detailed statistical comparison of the combined data sets as well as to place the level of metabolic variance in the context of that we have previously observed in tomato. To achieve the first of these goals we performed principal component analysis (PCA) and hierarchical clustering
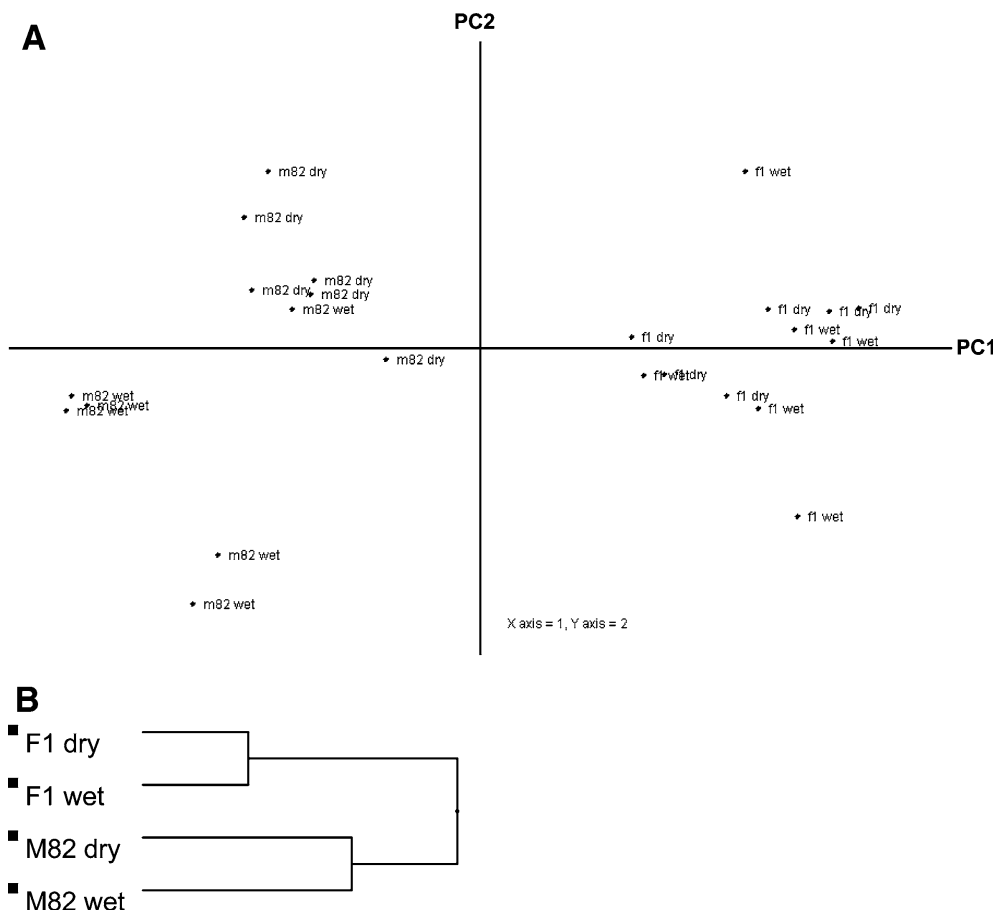

Figure 4. (A) Principal component analysis of irrigated (wet) and non-irrigated (dry) genotypes. The analysis was performed as defined in the Materials and Methods. Principle Component 1 (PC1) contributed with 55.2\% and Principle Component 2 (PC2) with 11.5\% to the separation. Major PC1 loadings were sucrose, fructose, glucose, phenylalanine, glutamine and asparagine. Major loadings of PC2 were gluconate, B-alanine, succinate and proline. (B) Hierarchical component analysis of M82 and F1 under wet and dry conditions, where the six replications of each treatment were averaged for each metabolite. 
analysis (HCA) on the entire dataset presented in the paper (figure 4A and B). The PCA analysis was able to clearly discriminate M82 from the F1 hybrid resulting from its cross with $S$. pennellii, as well as irrigated from non-irrigated M82 on the basis of the metabolite composition of their pericarp. However, PCA was not able to discriminate the irrigated from non-irrigated F1hybrid on this basis, even though statistical analysis at the 0.05 level could suggest that some of the differences observed statistically need to be interpreted with caution. In a similar vein, clustering by HCA revealed a strong influence of genotype (and by implication a weaker influence of environment), on the metabolic profiles. Given that the F1hybrid also displayed proportionally much less yield deficit under water stress and the fact that point-by-point analysis indicates the metabolite composition is similar in the irrigated hybrid to the non-irrigated M82 suggest that the hybrid may be "metabolically primed" to withstand water stress. In addition to this finding, which may well be of use for future genetic approaches to improve water tolerance in tomato, the results of this study uncovered a range of metabolites responding to water stress that may themselves be rationale targets for improving tolerance to this stress via transgenic or classical breeding methodologies.

\section{Acknowledgements}

The authors would like to thank the BMBF for funding in the frame of a DIP grant (Deutsch-Israeli Project).

\section{Electronic supplementary material}

The online version of this article (doi:10.1007/s11306007-0055-5) contains supplementary material, which is available to authorized users.

\section{References}

Avelange-Macherel, M.H., Ly-Vu, B., Delaunay, J., Richomme, P. and Leprince, O. (2006). NMR metabolite profiling analysis reveals changes in phospholipid metabolism associated with the re-establishment of dessication tolerance upon osmotic stress in germinated radicles of cucumber. Plant Cell Environ. 29, 471482.

Bartels, D. and Sunkar, R. (2005). Drought and salt tolerance in plants. Crit. Rev. Plant Sci. 24, 23-58.

Bohnert, H.J., Gong, Q., Li, P. and Ma, S. (2006). Unravelling abiotic stress tolerance mechanisms - getting genomics going. Curr. Opin. Plant Biol. 9, 180-188.

Büssis, D. and Heineke, D. (1998). Acclimation of potato plants to polyethylene glycol-induced water defecit II. Contents and subcellular distribution of organic solvents. J. Exp. Bot. 325, 1361-1370.

Broadhurst, D.I. and Kell, D.B. (2006). Statistical strategies for avoiding false discoveries in metabolomics and related experiments. Metabolomics 2, 179-196.
Dancer, J., David, M. and Stitt, M. (1990). Water stress leads to a change of partitioning in favour of sucrose in heterotrophic cell suspension cultures of Chenopodium rubrum. Plant Cell Environ. 13, 957-963.

Dumville, J.C. and Fry, S.C. (2002). Gentiobiose: a novel oligosaccharin in ripening tomato fruit. Planta 216, 484-495.

Eshed, Y. and Zamir, D. (1994). A genomic library of Lycopersicon pennellii in Lycopersicon esculentum - a tool for fine mapping of genes. Euphytica 79, 175-179.

Eshed, Y. and Zamir, D. (1995). An introgression line population of Lycopersicon pennellii in the cultivated tomato enables the identification and fine mapping of yield-associated QTL. Genetics 141, 1147-1162.

Fernie, A.R., Carrari, F. and Sweetlove, L.J. (2004). Respiratory metabolism: glycolysis, the TCA cycle and mitochondrial electron transport. Curr. Opin. Plant Biol. 7, 254-261.

Fridman, E., Liu, Y.S., Carmel-Goren, L., Gur, A., Shoresh, M., Pleban, T., Eshed, Y. and Zamir, D. (2002). Two tightly linked QTLs modify tomato sugar content via different physiological pathways. Mol. Gen. Gent. 266, 821-826.

Gong, Q.Q., Li, P.H., Ma, S.S., Rupassara, S.I. and Bohnert, H.J. (2005). Salinity stress adaptation competence in the extremophile Thellungiella halophila in comparison with its relative Arabidopsis thaliana. Plant J. 44, 826-839.

Granier, C., Aguirrezabal, L., Chenu, K., Cookson, S.J., Dauzat, M., Hamard, P., Thioux, J.-J., Rolland, G., Bouchier-Combaud, S., Lebaudy, A., Muller, B., Simonneau, T. and Tardieu, F. (2005). PHENOPSIS, an automated platform for reproducible phenotyping of plant responses to soil water deficit in Arabidopsis thaliana permitted the identification of an accession with low sensitivity to soil water deficit. New Phytol. 169, 623-635.

Gur, A. and Zamir, D. (2004). Unusual natural variation can lift yield barriers. PLOS Biol. 2, 1610-1615.

Hsieh, T.H., Lee, J.T., Charng, Y.Y. and Chan, M.T. (2002). Tomato plants ectopically expressing Arabidopsis CBF1 show enhanced resistance to water deficit stress. Plant Physiol. 130, 618-626.

Holtan, H.E.E. and Hake, S. (2003). Quantitative trait locus analysis of leaf dissection in tomato using Lycopersicon pennellii segmental introgression lines. Genetics 165, 1541-1550.

Ishizaki, K., Larson, T.R., Schauer, N., Fernie, A.R., Graham, I.A. and Leaver, C.J. (2005). The critical role of Arabidopsis electron-transfer flavoprotein: Ubiqinone oxidoreductase during dark-induced starvation. Plant Cell 9, 2587-2600.

Kalifa, Y., Perlson, E., Gilad, A., Konrad, Z., Scolnik, P.A. and BarZvi, D. (2004). Over-expression of the water and salt stressregulated Asr1 gene confers an increased salt tolerance. Plant Cell Environ. 27, 1459-1468.

Kaplan, F., Kopka, J., Haskell, D.W., Zhao, W., Schiller, K.C., Gatzke, N., Sung, D.Y. and Guy, C.L. (2004). Exploring the temperature-stress metabolome of Arabidopsis. Plant Physiol. 136, 4159-4168.

Kopka, J., Schauer, N., Kruger, S., Birkemeyer, C., Usadel, B., Bergmüller, E., Dörmann, P., Gibon, Y., Stitt, M., Willmitzer, L., Fernie, A.R. and Steinhauser, D. (2005). GMD@CSB.DB: The Golm Metabolome Database. Bioinformatics 21, 16351638.

Liu, Y.S., Gur, A., Ronen, G., Causse, M., Damidaux, R., Buret, M., Hirschberg, J. and Zamir, D (2003). There is more to tomato colour than carotenoid genes. Plant Biot. J. 1, 195-207.

Mittler, R. (2006). Abiotic stress, the field environment and stress combination. Trends Plant Sci. 11, 15-19.

Rhoades, D. and Hanson, A.D. (1993). Quaternary ammonium and tertiary sulfonium compounds in higher plants. Annu. Rev. Plant Physiol. Plant Mol. Biol. 44, 357-384.

Roessner, U., Wagner, C., Kopka, J., Trethewey, R.N. and Willmitzer, L. (2000). Simultaneous analysis of metabolites in potato tuber by gas-chromatography mass-spectrometry. Plant J. 23, $131-142$. 
Roessner, U., Luedemann, A., Brust, D., Fiehn, O., Linke, T., Willmitzer, L. and Fernie, A.R. (2001). Metabolic profiling allows comprehensive phenotyping of genetically or environmentally modified plant systems. Plant Cell 13, 11-29.

Roessner, U., Patterson, J.H., Forbes, M.G., Fincher, G.B., Langridge, P. and Bacic, A. (2006). An investigation of boron toxicity in barley using metabolomics. Plant Physiol. 142, 447-454.

Roessner-Tunali, U., Hegemann, B., Lytovchenko, A., Carrari, F., Bruedigam, C., Granot, D. and Fernie, A.R. (2003). Metabolic profiling of transgenic tomato plants overexpressing hexokinase reveals that the influence of hexose phosphorylation diminishes during fruit development. Plant Physiol. 133, 84-99.

Saeed, A.I., Sharov, V., White, J., Li, J., Liang, W., Bhagabati, N., Braisted, J., Klapa, M., Currier, T., Thiagarajan, M., Sturn, A., Snuffin, M., Rezantsev, A., Popov, D., Ryltsov, A., Kostukovich, E., Borisovsky, I., Liu, Z., Vinsavich, A., Trush, V. and Quackenbush, J. (2003). TM4: a free, open-source system for microarray data management and analysis. Biotechniques $\mathbf{3 4}$ 374-378.

Schauer, N., Zamir, D. and Fernie, A.R (2005). Metabolic profiling of leaves and fruit of wild species tomato: a survey of the Solanum lycopersicum complex. J. Exp. Bot. 56, 297-307.

Schauer, N., Steinhauser, D., Strelkov, S., Schomburg, D., Allison, G., Moritz, T., Lundgren, K., Roessner-Tunali, U., Forbes, M.G., Willmitzer, L., Fernie, A.R. and Kopka, J. (2005). GC-MS libraries for the rapid identification of metabolites in complex biological samples. FEBS Lett. 579, 1332-1337.

Schauer, N., Semel, Y., Roessner, U., Gur, A., Balbo, I., Carrari, F., Pleban, T., Perez-Melis, A., Bruedigam, C., Kopka, J., Willm- itzer, L., Zamir, D. and Fernie, A.R. (2006). Genetics of metabolite content in fruits of interspecific introgressions of tomato. Nature Biotechnol. 24, 447-454.

Semel, Y., Nissenbaum, J., Menda, N., Zinder, M., Krieger, U., Issman, N. and Zamir, D. (2006). Overdominant quantitative trait loci for yield and fitness in tomato. Proc. Natl. Acad. Sci. U.S.A. 103, 12981-12986.

Theilhaber, J., Connolly, T., Roman-Roman, S., Bushnell, S., Jackson, A., Call, K. and Garcia Baron, T. R. (2002). Finding Genes in the $\mathrm{C} 2 \mathrm{Cl} 2$ Osteogenic Pathway by KNearest-Neighbor Classification of Expression Data. Genome Res. 12, 165-176.

Urbanczyk-Wochniak, E. and Fernie, A.R. (2005). Metabolic profiling reveals altered nitrogen nutrient regimes have diverse effects on the metabolism of hydroponically-grown tomato (Solanum lycopersicum) plants. J. Exp. Bot. 56, 309-321.

Yamaguchi-Shinozaki, K. and Shinozaki, K. (2006). Transcriptional regulatory networks in cellular responses and tolerance to dehydration and cold stresses. Annu. Rev. Plant Biol. 57, 781893.

Yoshiba, Y., Kiyosue, T., Nakashima, K., Yamaguchi-Shinozaki, K. and Shinozaki, K. (1997). Regulation of levels of proline as an osmolyte in plants under water stress. Plant Cell Physiol. 38, 1095-1102.

Zamir, D. (2001). Improving plant breeding with exotic genetic libraries. Nat. Rev. Gen. 12, 983-989.

Zhu, J.K. (2000). Genetic analysis of plant salt tolerance using Arabidopsis. Plant Physiol. 124, 941-948. 